\title{
gs \\ Magnetization dissipation in ferromagnets from scattering theory
}

\author{
Arne Brataas* \\ Department of Physics, Norwegian University of Science and Technology, NO-7491 Trondheim, Norway \\ Yaroslav Tserkovnyak \\ Department of Physics and Astronomy, University of California, Los Angeles, California 90095, USA
}

Gerrit E. W. Bauer

Institute for Materials Research, Tohoku University, Sendai 980-8577, Japan and Kavli Institute of NanoScience, Delft University of Technology, Lorentzweg 1, 2628 CJ Delft, The Netherlands

(Received 8 April 2011; published 5 August 2011)

\begin{abstract}
The magnetization dynamics of ferromagnets is often formulated in terms of the Landau-Lifshitz-Gilbert (LLG) equation. The reactive part of this equation describes the response of the magnetization in terms of effective fields, whereas the dissipative part is parametrized by the Gilbert damping tensor. We formulate a scattering theory for the magnetization dynamics and map this description on the linearized LLG equation by attaching electric contacts to the ferromagnet. The reactive part can then be expressed in terms of the static scattering matrix. The dissipative contribution to the low-frequency magnetization dynamics can be described as an adiabatic energy pumping process to the electronic subsystem by the time-dependent magnetization. The Gilbert damping tensor depends on the time derivative of the scattering matrix as a function of the magnetization direction. By the fluctuation-dissipation theorem, the fluctuations of the effective fields can also be formulated in terms of the quasistatic scattering matrix. The theory is formulated for general magnetization textures and worked out for monodomain precessions and domain-wall motions. We prove that the Gilbert damping from scattering theory is identical to the result obtained by the Kubo formalism.
\end{abstract}

DOI: 10.1103/PhysRevB.84.054416

PACS number(s): 75.40.Gb, 76.60.Es, 72.25.Mk

\section{INTRODUCTION}

Ferromagnets develop a spontaneous magnetization below the Curie temperature. The long-wavelength modulations of the magnetization direction consist of spin waves, the lowlying elementary excitations (Goldstone modes) of the ordered state. When the thermal energy is much smaller than the microscopic exchange energy, the magnetization dynamics can be phenomenologically expressed in a generalized LandauLifshitz-Gilbert (LLG) form:

$$
\begin{aligned}
\dot{\mathbf{m}}(\mathbf{r}, t)= & -\gamma \mathbf{m}(\mathbf{r}, t) \times\left[\mathbf{H}_{\mathrm{eff}}(\mathbf{r}, t)+\mathbf{h}(\mathbf{r}, t)\right] \\
& +\mathbf{m}(\mathbf{r}, t) \times \int d \mathbf{r}^{\prime}\left[\tilde{\alpha}[\mathbf{m}]\left(\mathbf{r}, \mathbf{r}^{\prime}\right) \dot{\mathbf{m}}\left(\mathbf{r}^{\prime}, t\right)\right],
\end{aligned}
$$

where the magnetization texture is described by $\mathbf{m}(\mathbf{r}, t)$, the unit vector along the magnetization direction at position $\mathbf{r}$ and time $t, \dot{\mathbf{m}}(\mathbf{r}, t)=\partial \mathbf{m}(\mathbf{r}, t) / \partial t$, and $\gamma=g \mu_{B} / \hbar$ is the gyromagnetic ratio in terms of the $g$ factor $(\approx 2$ for free electrons) and the Bohr magneton $\mu_{B}$. The Gilbert damping $\tilde{\alpha}$ is a nonlocal symmetric $3 \times 3$ tensor that is a functional of m. The Gilbert damping tensor is commonly approximated to be diagonal and isotropic (i), local (1), and independent of the magnetization $\mathbf{m}$, with diagonal elements

$$
\alpha_{\mathrm{il}}\left(\mathbf{r}, \mathbf{r}^{\prime}\right)=\alpha \delta\left(\mathbf{r}-\mathbf{r}^{\prime}\right) .
$$

The linearized version of the LLG equation for smallamplitude excitations has been derived microscopically. ${ }^{1}$ It has been used very successfully to describe the measured response of ferromagnetic bulk materials and thin films in terms of a small number of adjustable, material-specific parameters. The experiment of choice is ferromagnetic resonance (FMR), which probes the small-amplitude coherent precession of the magnet. ${ }^{2}$ The Gilbert damping model in the local and time-independent approximation has important ramifications, such as a linear dependence of the FMR linewidth on resonance frequency, that have been frequently found to be correct. The damping constant is technologically important since it governs the switching rate of ferromagnets driven by external magnetic fields or electric currents. ${ }^{3}$ In spatially dependent magnetization textures, the nonlocal character of the damping can be significant as well. ${ }^{4-6}$ Motivated by the belief that the Gilbert damping constant is an important material property, we set out here to understand its physical origins from first principles. We focus on the well-studied and technologically important itinerant ferromagnets, although the formalism can be used in principle for any magnetic system.

The reactive dynamics within the LLG Eq. (1) is described by the thermodynamic potential $\Omega[\mathbf{M}]$ as a functional of the magnetization. The effective magnetic field $\mathbf{H}_{\mathrm{eff}}[\mathbf{M}](\mathbf{r}) \equiv$ $-\delta \Omega / \delta \mathbf{M}(\mathbf{r})$ is the functional derivative with respect to the local magnetization $\mathbf{M}(\mathbf{r})=M_{s} \mathbf{m}(r)$, including the external magnetic field $\mathbf{H}_{\text {ext }}$, the magnetic dipolar field $\mathbf{H}_{d}$, the texturedependent exchange energy, and crystal-field anisotropies. $M_{S}$ is the saturation magnetization density. Thermal fluctuations can be included by a stochastic magnetic field $\mathbf{h}(\mathbf{r}, t)$ with zero time average, $\langle\mathbf{h}\rangle=0$, and white-noise correlation: ${ }^{7}$

$$
\left\langle h_{i}(\mathbf{r}, t) h_{j}\left(\mathbf{r}^{\prime}, t^{\prime}\right)\right\rangle=\frac{2 k_{B} T}{\gamma M_{s}} \tilde{\alpha}_{i j}[\mathbf{m}]\left(\mathbf{r}, \mathbf{r}^{\prime}\right) \delta\left(t-t^{\prime}\right),
$$

where $M_{s}$ is the magnetization, $i$ and $j$ are the Cartesian indices, and $T$ is the temperature. This relation is a consequence of the fluctuation-dissipation theorem (FDT) in the classical (Maxwell-Boltzmann) limit. 


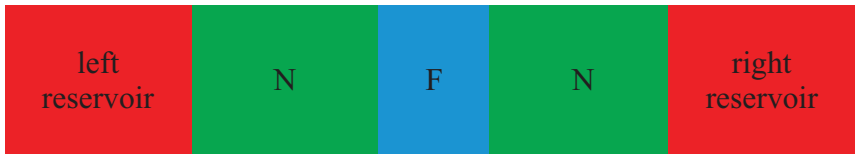

FIG. 1. (Color online) Schematic picture of a ferromagnet $(F)$ in contact with a thermal bath (reservoirs) via metallic normal metal leads $(N)$.

The scattering $(S)$ matrix is defined in the space of the transport channels that connect a scattering region (the sample) to real or fictitious thermodynamic (left and right) reservoirs by electric contacts with leads that are modeled as ideal waveguides. Scattering matrices are known to describe transport properties, such as the giant magnetoresistance, spin pumping, and current-induced magnetization dynamics in layered normal-metal $(N) \mid$ ferromagnet $(F) .{ }^{8-10}$ When the ferromagnet is part of an open system as in Fig. $1, \Omega$ can also be expressed in terms of the scattering matrix, which has been used to express the nonlocal exchange coupling between ferromagnetic layers through conducting spacers. ${ }^{11}$ We will show here that the scattering matrix description of the effective magnetic fields is valid even when the system is closed, provided the dominant contribution comes from the electronic band structure, scattering potential disorder, and spin-orbit interaction.

Scattering theory can also be used to compute the Gilbert damping tensor $\tilde{\alpha}$ for magnetization dynamics. ${ }^{12}$ The energyloss rate of the scattering region can be expressed in terms of the time-dependent $S$ matrix. To this end, the theory of adiabatic quantum pumping has to be generalized to describe dissipation in a metallic ferromagnet. The Gilbert damping tensor is found by evaluating the energy pumping out of the ferromagnet and relating it to the energy loss that is dictated by the LLG equation. In this way, it is proven that the Gilbert phenomenology is valid beyond the linearresponse regime of small magnetization amplitudes. The key approximation that is necessary to derive Eq. (1) including $\tilde{\alpha}$ is the (adiabatic) assumption that the ferromagnetic resonance frequency $\omega_{\text {FMR }}$ that characterizes the magnetization dynamics is small compared to the internal energy scale set by the exchange splitting $\Delta$ and spin-flip relaxation rates $\tau_{s}$. The LLG phenomenology works well for ferromagnets for which $\omega_{\text {FMR }} \ll \Delta / \hbar$, which is certainly the case for transition-metal ferromagnets such as Fe and Co.

Gilbert damping in transition-metal ferromagnets is generally believed to stem from the transfer of energy from the magnetic order parameter to the itinerant quasiparticle continuum. This requires either magnetic disorder or spin-orbit interactions in combination with impurity and/or phonon scattering. ${ }^{2}$ Since the heat capacitance of the ferromagnet is dominated by the lattice, the energy transferred to the quasiparticles will be dissipated to the lattice as heat. Here we focus on the limit in which elastic scattering dominates, such that the details of the heat transfer to the lattice do not affect our results. Our approach formally breaks down in sufficiently clean samples at high temperatures in which inelastic electron-phonon scattering dominates. Nevertheless, quantitative insight can be gained by our method even in that limit by modeling phonons by frozen deformations. ${ }^{13}$
In the present formulation, the heat generated by the magnetization dynamics can escape only via the contacts to the electronic reservoirs. By computing this heat current through the contacts, we access the total dissipation rate. Part of the heat and spin current that escapes the sample is due to spin pumping, which causes energy and momentum loss even for otherwise dissipationless magnetization dynamics. This process is now well understood. ${ }^{10,14,15}$ For sufficiently large samples, the spin-pumping contribution is overwhelmed by the dissipation in the bulk of the ferromagnet. Both contributions can be separated by studying the heat generation as a function of the length of a wire. In principle, a voltage can be added to study dissipation in the presence of electric currents, as in Refs. 16 and 17, but we concentrate here on a common and constant chemical potential in both reservoirs. The spin-orbit interaction or the interaction with magnetic impurities (or with scalar impurities in the presence of crystalline exchange field inhomogeneities) enters our formalism via the scattering matrices. When these spin interactions are set to zero, the application of our formulation will only give magnetization dissipation caused by spin pumping, in accordance with the conservation of the total spin angular momentum in a closed system (which is valid when neglecting all relativistic corrections, both at the atomistic and band-structure levels).

Although it is not a necessity, results can be simplified by expanding the $S$ matrix to lowest order in the amplitude of the magnetization dynamics. In this limit, scattering theory and the Kubo linear-response formalism for the dissipation can be directly compared. We will demonstrate explicitly that both approaches lead to identical results, which increases our confidence in our method. The coupling to the reservoirs of large samples is identified to play the same role as the infinitesimals in the Kubo approach, which guarantee causality.

Our formalism was introduced first in Ref. 12 and was limited to the macrospin model and zero temperature. An extension to the friction associated with domain-wall motion was given in Ref. 16. Here we show how to handle general magnetization textures and finite temperatures. Furthermore, we offer an alternative route to derive the Gilbert damping in terms of the scattering matrix from the thermal fluctuations of the effective field. We also explain in more detail the relation of the present theory to spin and charge pumping by magnetization textures.

The Gilbert damping has been derived microscopically from the Kubo formalism. ${ }^{1}$ This formulation has been implemented by first-principles electronic band structures. ${ }^{18}$ However, the simplest $a b$ initio implementation of the Kubo approach requires additional approximations such as the relaxation-time approximation and the neglect of disorder vertex corrections. An advantage of the scattering theory of Gilbert damping is its suitability for modern $a b$ initio techniques of spin transport that do not suffer from these drawbacks. ${ }^{12,19}$ The scattering theory of magnetization dissipation can also be equivalently formulated in a KuboGreenwood Green's function form that can be evaluated. ${ }^{12,20}$

Our paper is organized in the following way. In Sec. II, we introduce our microscopic model for the ferromagnet. In Sec. III, dissipation in the Landau-Lifshitz-Gilbert equation is exposed. The scattering theory of magnetization dynamics is developed in Sec. IV. We discuss the Kubo formalism for the 
time-dependent magnetizations in Sec. V, before concluding our paper in Sec. VI. The Appendixes provide technical derivations of spin, charge, and energy pumping in terms of the scattering matrix of the system.

\section{MODEL}

Our approach rests on density-functional theory (DFT), which is widely and successfully used to describe the electronic structure and magnetism in many ferromagnets, including transition-metal ferromagnets and ferromagnetic semiconductors. ${ }^{21}$ In the Kohn-Sham implementation of DFT, noninteracting hypothetical particles experience an effective exchange-correlation potential that leads to the same groundstate density as the interacting many-electron system. ${ }^{22} \mathrm{~A}$ simple yet successful scheme is the local-density approximation to the effective potential. DFT theory can also handle time-dependent phenomena. We adopt here the adiabatic localdensity approximation (ALDA), i.e., an exchange-correlation potential that is time-dependent but local in time and space. ${ }^{23,24}$ As the name expresses, the ALDA is valid when the parametric time dependence of the problem is adiabatic with respect to the electron time constants. Here we consider a magnetization direction that varies slowly in both space and time. The ALDA should be suited to treat magnetization dynamics, since the typical time scale $\left[t_{\mathrm{FMR}} \sim 1 /(10 \mathrm{GHz}) \sim 10^{-10} \mathrm{~s}\right]$ is long compared to that associated with the Fermi and exchange energies, $1-10 \mathrm{eV}$, leading to $\hbar / \Delta \sim 10^{-13} \mathrm{~s}$ in transition-metal ferromagnets.

In the ALDA, the system is described by the time-dependent effective Schrödinger equation

$$
\hat{H}_{\mathrm{ALDA}} \Psi(\mathbf{r}, t)=i \hbar \frac{\partial}{\partial t} \Psi(\mathbf{r}, t),
$$

where $\Psi(\mathbf{r}, t)$ is the quasiparticle wave function at position $\mathbf{r}$ and time $t$. We consider a generic mean-field electronic Hamiltonian that depends on the magnetization direction $\hat{H}_{\text {ALDA }}[\mathbf{m}]$ and includes the periodic Hartree, exchange, and correlation potentials and relativistic corrections such as the spin-orbit interaction. Impurity scattering including magnetic disorder is also represented by $\hat{H}_{\text {ALDA }}$. The magnetization $\mathbf{m}$ is allowed to vary in time and space. The total Hamiltonian depends additionally on the Zeeman energy of the magnetization in external $\mathbf{H}_{\mathrm{ext}}$ and dipolar $\mathbf{H}_{d}$ magnetic fields:

$$
\hat{H}=\hat{H}_{\mathrm{ALDA}}[\mathbf{m}]-M_{s} \int d r \mathbf{m} \cdot\left(\mathbf{H}_{\mathrm{ext}}+\mathbf{H}_{d}\right) .
$$

For this general Hamiltonian (5), our task is to deduce an expression for the Gilbert damping tensor $\tilde{\alpha}$. To this end, from the form of the Landau-Lifshitz-Gilbert equation (3), it is clear that we should seek an expansion in terms of the slow variations of the magnetizations in time. Such an expansion is valid provided the adiabatic magnetization precession frequency is much less than the exchange splitting $\Delta$ or the spin-orbit energy that governs spin relaxation of electrons. We discuss first the dissipation in the LLG equation and subsequently compare it with the expressions from scattering theory of electron transport. This leads to a recipe to describe dissipation by first principles. Finally, we discuss the connection to the Kubo linear-response formalism and prove that the two formulations are identical in linear response.

\section{DISSIPATION AND LANDAU-LIFSHITZ-GILBERT EQUATION}

The energy dissipation can be obtained from the solution of the LLG Eq. (1) as

$$
\begin{aligned}
\dot{E} & =-M_{s} \int d \mathbf{r}\left[\dot{\mathbf{m}}(\mathbf{r}, t) \cdot \mathbf{H}_{\mathrm{eff}}(\mathbf{r}, t)\right] \\
& =-\frac{M_{s}}{\gamma} \int d \mathbf{r} \int d \mathbf{r}^{\prime} \dot{\mathbf{m}}(\mathbf{r}) \cdot \tilde{\alpha}[\mathbf{m}]\left(\mathbf{r}, \mathbf{r}^{\prime}\right) \cdot \dot{\mathbf{m}}\left(\mathbf{r}^{\prime}\right) .
\end{aligned}
$$

The scattering theory of magnetization dissipation can be formulated for arbitrary spatiotemporal magnetization textures. Much insight can be gained for certain special cases. In small particles or high magnetic fields, the collective magnetization motion is approximately constant in space and the "macrospin" model is valid in which all spatial dependences are disregarded. We will also consider special magnetization textures with a dynamics characterized by a number of dynamic (soft) collective coordinates $\xi_{a}(t)$ counted by $a:^{25,26}$

$$
\mathbf{m}(\mathbf{r}, t)=\mathbf{m}_{\mathrm{st}}\left(\mathbf{r} ;\left\{\xi_{a}(t)\right\}\right),
$$

where $\mathbf{m}_{\mathrm{st}}$ is the profile at $t \rightarrow-\infty$. This representation has proven to be very effective in handling magnetization dynamics of domain walls in ferromagnetic wires. The description is approximate, but (for a few variables) it becomes exact in special limits, such as a transverse domain wall in wires below the Walker breakdown (see below); it becomes arbitrarily accurate by increasing the number of collective variables. The energy dissipation to lowest (quadratic) order in the rate of change $\dot{\xi}_{a}$ of the collective coordinates is

$$
\dot{E}=-\sum_{a b} \tilde{\Gamma}_{a b} \dot{\xi}_{a} \dot{\xi}_{b}
$$

The (symmetric) dissipation tensor $\tilde{\Gamma}_{a b}$ reads

$$
\tilde{\Gamma}_{a b}=\frac{M_{s}}{\gamma} \int d \mathbf{r} \int d \mathbf{r}^{\prime} \frac{\partial \mathbf{m}_{\mathrm{st}}(\mathbf{r})}{\partial \xi_{a}} \alpha[\mathbf{m}]\left(\mathbf{r}, \mathbf{r}^{\prime}\right) \cdot \frac{\partial \mathbf{m}_{\mathrm{st}}\left(\mathbf{r}^{\prime}\right)}{\partial \xi_{b}} .
$$

The equation of motion of the collective coordinates under a force

$$
\mathfrak{F}=-\frac{\partial \Omega}{\partial \xi}
$$

is $^{25,26}$

$$
\tilde{\eta} \dot{\xi}+[\mathfrak{F}+\mathfrak{f}(t)]-\tilde{\Gamma} \dot{\xi}=0,
$$

introducing the antisymmetric and time-independent gyrotropic tensor:

$$
\tilde{\eta}_{a b}=\frac{M_{s}}{\gamma} \int d \mathbf{r} \mathbf{m}_{\mathrm{st}}(\mathbf{r}) \cdot\left[\frac{\partial \mathbf{m}_{\mathrm{st}}(\mathbf{r})}{\partial \xi_{a}} \times \frac{\partial \mathbf{m}_{\mathrm{st}}(\mathbf{r})}{\partial \xi_{b}}\right] .
$$

We show below that $\mathfrak{F}$ and $\tilde{\Gamma}$ can be expressed in terms of the scattering matrix. For our subsequent discussions, it is necessary to include a fluctuating force $\mathfrak{f}(t)$ [with $\langle\mathfrak{f}(t)\rangle=0$ ], which has not been considered in Refs. 25 and 26. From Eq. (3) 
if follows that the time correlation of $\mathfrak{f}$ is white and obeys the fluctuation-dissipation theorem:

$$
\left\langle\mathfrak{f}_{a}(t) \mathfrak{f}_{b}\left(t^{\prime}\right)\right\rangle=2 k_{B} T \tilde{\Gamma}_{a b} \delta\left(t-t^{\prime}\right) .
$$

In the following, we illustrate the collective coordinate description of magnetization textures for the macrospin model and the Walker model for a transverse domain wall. The treatment is easily extended to other rigid textures such as magnetic vortices.

\section{A. Macrospin excitations}

When high magnetic fields are applied or when the system dimensions are small, the exchange stiffness dominates. In both limits, the magnetization direction and its low-energy excitations lie on the unit sphere and its magnetization dynamics is described by the polar angles $\theta(t)$ and $\varphi(t)$ :

$$
\mathbf{m}=(\sin \theta \cos \varphi, \sin \theta \sin \varphi, \cos \theta) .
$$

The diagonal components of the gyrotropic tensor vanish by (anti)symmetry $\tilde{\eta}_{\theta \theta}=0, \tilde{\eta}_{\varphi \varphi}=0$. Its off-diagonal components are

$$
\eta_{\theta \varphi}=\frac{M_{s} V}{\gamma} \sin \theta=-\eta_{\varphi \theta} .
$$

$V$ is the particle volume and $M_{s} V$ is the total magnetic moment. We now have two coupled equations of motion,

$$
\begin{gathered}
\frac{M_{s} V}{\gamma} \dot{\varphi} \sin \theta-\frac{\partial \Omega}{\partial \theta}-\left(\tilde{\Gamma}_{\theta \theta} \dot{\theta}+\tilde{\Gamma}_{\theta \varphi} \dot{\varphi}\right)=0, \\
-\frac{M_{s} V}{\gamma} \dot{\theta} \sin \theta-\frac{\partial \Omega}{\partial \varphi}-\left(\tilde{\Gamma}_{\varphi \theta} \dot{\theta}+\tilde{\Gamma}_{\varphi \varphi} \dot{\varphi}\right)=0 .
\end{gathered}
$$

The thermodynamic potential $\Omega$ determines the ballistic trajectories of the magnetization. The Gilbert damping tensor $\tilde{\Gamma}_{a b}$ will be computed below, but when isotropic and local,

$$
\tilde{\Gamma}=\tilde{1} \delta\left(\mathbf{r}-\mathbf{r}^{\prime}\right) M_{s} \alpha / \gamma,
$$

where $\tilde{1}$ is a unit matrix in the Cartesian basis and $\alpha$ is the dimensionless Gilbert constant, $\Gamma_{\theta \theta}=M_{s} V \alpha / \gamma, \Gamma_{\theta \varphi}=0=$ $\Gamma_{\varphi \theta}$, and $\Gamma_{\varphi \varphi}=\sin ^{2} \theta M_{s} V \alpha / \gamma$.

\section{B. Domain-wall motion}

We focus on a one-dimensional model, in which the magnetization gradient, magnetic easy axis, and external magnetic field point along the wire $(z)$ axis. The magnetic energy of such a wire with transverse cross section $S$ can be written as $^{27}$

$$
\Omega=M_{s} S \int d z \phi(z),
$$

in terms of the one-dimensional energy density

$$
\phi=\frac{A}{2}\left|\frac{\partial \mathbf{m}}{\partial z}\right|^{2}-H_{a} m_{z}+\frac{K_{1}}{2}\left(1-m_{z}^{2}\right)+\frac{K_{2}}{2} m_{x}^{2},
$$

where $H_{a}$ is the applied field and $A$ is the exchange stiffness. Here the easy-axis anisotropy is parametrized by an anisotropy constant $K_{1}$. In the case of a thin-film wire, there is also a smaller anisotropy energy associated with the magnetization transverse to the wire governed by $K_{2}$. In a cylindrical wire from a material without crystal anisotropy (such as permalloy), $K_{2}=0$.

When the shape of such a domain wall is preserved in the dynamics, three collective coordinates characterize the magnetization texture: the domain-wall position $\xi_{1}(t)=$ $r_{\mathrm{w}}(t)$, the polar angle $\xi_{2}(t)=\varphi_{\mathrm{w}}(t)$, and the domain-wall width $\lambda_{\mathrm{w}}(t)$. We consider a head-to-head transverse domain wall (a tail-to-tail wall can be treated analogously). $\mathbf{m}(z)=$ $\left(\sin \theta_{\mathrm{w}} \cos \varphi_{\mathrm{w}}, \sin \theta_{\mathrm{w}} \sin \varphi_{\mathrm{w}}, \cos \theta_{\mathrm{w}}\right)$, where

$$
\cos \theta_{\mathrm{w}}=\tanh \frac{r_{\mathrm{w}}-z}{\lambda_{\mathrm{w}}}
$$

or, represented as

$$
\csc \theta_{\mathrm{w}}=\cosh \frac{r_{\mathrm{w}}-z}{\lambda_{\mathrm{w}}},
$$

minimizes the energy (20) under the constraint that the magnetization to the far left and right points toward the domain wall. The off-diagonal elements are then $\tilde{\eta}_{r l}=0=\tilde{\eta}_{l r}$ and $\tilde{\eta}_{r \varphi}=-2 M_{s} / \gamma=-\tilde{\eta}_{\varphi r}$. The energy (20) reduces to

$$
\Omega=M_{s} S\left[A / \lambda_{\mathrm{w}}-2 H_{a} r+K_{1} \lambda_{\mathrm{w}}+K_{2} \lambda_{\mathrm{w}} \cos ^{2} \varphi_{\mathrm{w}}\right] .
$$

Disregarding fluctuations, the equation of motion Eq. (12) can be expanded as

$$
\begin{gathered}
2 \dot{r}_{\mathrm{w}}+\alpha_{\varphi \varphi} \dot{\varphi}+\alpha_{\varphi r} \dot{r}_{\mathrm{w}}+\alpha_{\varphi \lambda} \dot{\lambda}_{\mathrm{w}}=\gamma K_{2} \lambda_{\mathrm{w}} \sin 2 \varphi_{\mathrm{w}}, \\
-2 \dot{\varphi}+\alpha_{r r} \dot{r}_{\mathrm{w}}+\alpha_{r \varphi} \dot{\varphi}+\alpha_{r \lambda} \dot{\lambda}_{\mathrm{w}}=2 \gamma H_{a}, \\
A / \lambda_{\mathrm{w}}^{2}+\alpha_{\lambda r} \dot{r}_{\mathrm{w}}+\alpha_{\lambda \varphi} \dot{\varphi}+\alpha_{\lambda \lambda} \dot{\lambda}_{\mathrm{w}}=K_{1}+K_{2} \cos ^{2} \varphi_{\mathrm{w}},
\end{gathered}
$$

where $\alpha_{a b}=\gamma \Gamma_{a b} / M_{s} S$.

When the Gilbert damping tensor is isotropic and local in the basis of the Cartesian coordinates, $\tilde{\Gamma}=\tilde{1} \delta\left(\mathbf{r}-\mathbf{r}^{\prime}\right) M_{s} \alpha / \gamma$,

$$
\alpha_{r r}=\frac{2 \alpha}{\lambda_{\mathrm{w}}}, \quad \alpha_{\varphi \varphi}=2 \alpha \lambda_{\mathrm{w}}, \quad \alpha_{\lambda \lambda}=\frac{\pi^{2} \alpha}{6 \lambda_{\mathrm{w}}},
$$

whereas all off-diagonal elements vanish.

Most experiments are carried out on thin-film ferromagnetic wires for which $K_{2}$ is finite. Dissipation is especially simple below the Walker threshold, the regime in which the wall moves with a constant drift velocity, $\dot{\varphi}_{\mathrm{w}}=0$, and ${ }^{28}$

$$
\dot{r}_{\mathrm{w}}=-2 \gamma H_{a} / \alpha_{r r} .
$$

The Gilbert damping coefficient $\alpha_{r r}$ can be obtained directly from the scattering matrix by the parametric dependence of the scattering matrix on the center coordinate position $r_{\mathrm{w}}$. When the Gilbert damping tensor is isotropic and local, we find $\dot{r}_{\mathrm{w}}=\lambda_{\mathrm{w}} \gamma H_{a} / \alpha$. The domainwall width $\lambda_{\mathrm{w}}=\sqrt{A /\left(K_{1}+K_{2} \cos ^{2} \varphi_{\mathrm{w}}\right)}$ and the out-of-plane angle $\varphi_{\mathrm{w}}=\frac{1}{2} \arcsin 2 \gamma H_{a} / \alpha K_{2}$. At the Walker-breakdown field $\left(H_{a}\right)_{\mathrm{WB}}=\alpha K_{2} /(2 \gamma)$, the sliding domain wall becomes unstable.

In a cylindrical wire without anisotropy, $K_{2}=0, \varphi_{\mathrm{w}}$ is time-dependent and satisfies

$$
\dot{\varphi}_{\mathrm{w}}=-\frac{\left(2+\alpha_{\varphi r}\right)}{\alpha_{\varphi \varphi}} \dot{r}_{\mathrm{w}}
$$

while

$$
\dot{r}_{\mathrm{w}}=\frac{2 \gamma H_{a}}{2\left(\frac{2+\alpha_{\varphi r}}{\alpha_{\varphi \varphi}}\right)+\alpha_{r r}} .
$$


For isotropic and local Gilbert damping coefficients, ${ }^{27}$

$$
\frac{\dot{r}_{\mathrm{w}}}{\lambda_{\mathrm{w}}}=\frac{\alpha \gamma H_{a}}{1+\alpha^{2}} .
$$

In the next section, we formulate how the Gilbert scattering tensor can be computed from time-dependent scattering theory.

\section{SCATTERING THEORY OF MESOSCOPIC MAGNETIZATION DYNAMICS}

Scattering theory of transport phenomena ${ }^{29}$ has proven its worth in the context of magnetoelectronics. It has been used advantageously to evaluate the nonlocal exchange interaction multilayers or spin valves, ${ }^{11}$ the giant magnetoresistance, ${ }^{30}$ spin-transfer torque, ${ }^{9}$ and spin pumping. ${ }^{10}$ We first review the scattering theory of equilibrium magnetic properties and anisotropy fields and then we will turn to nonequilibrium transport.

\section{A. Conservative forces}

Considering only the electronic degrees of freedom in our model, the thermodynamic (grand) potential is defined as

$$
\Omega=-k_{B} T \ln \operatorname{Tr} e^{-\left(\hat{H}_{\mathrm{ALDA}}-\mu \hat{N}\right)},
$$

while $\mu$ is the chemical potential and $\hat{N}$ is the number operator. The conservative force

$$
\mathfrak{F}=-\frac{\partial \Omega}{\partial \xi}
$$

can be computed for an open system by defining a scattering region that is connected by ideal leads to reservoirs at common equilibrium. For a two-terminal device, the flow of charge, spin, and energy between the reservoirs can then be described in terms of the $S$ matrix:

$$
S=\left(\begin{array}{ll}
\mathfrak{r} & \mathfrak{t}^{\prime} \\
\mathfrak{t} & \mathfrak{r}^{\prime}
\end{array}\right)
$$

where $\mathfrak{r}$ is the matrix of probability amplitudes of states impinging from and reflected into the left reservoir, while $t$ denotes the probability amplitudes of states incoming from the left and transmitted to the right. Similarly, $\mathfrak{r}^{\prime}$ and $\mathfrak{t}^{\prime}$ describe the probability amplitudes for states that originate from the right reservoir. $\mathfrak{r}, \mathfrak{r}^{\prime}, \mathfrak{t}$, and $\mathfrak{t}^{\prime}$ are matrices in the space spanned by eigenstates in the leads. We are interested in the free magnetic energy modulation by the magnetic configuration that allows evaluation of the forces Eq. (33). The free-energy change reads

$$
\Delta \Omega=-k_{B} T \int d \epsilon \Delta n(\epsilon) \ln \left[1+e^{(\epsilon-\mu) / k_{B} T}\right],
$$

where $\Delta n(\epsilon) d \epsilon$ is the change in the number of states at energy $\epsilon$ and interval $d \epsilon$, which can be expressed in terms of the scattering matrix ${ }^{31}$

$$
\Delta n(\epsilon)=-\frac{1}{2 \pi i} \frac{\partial}{\partial \epsilon} \operatorname{Tr} \ln S(\epsilon) .
$$

Carrying out the derivative, we arrive at the force

$$
\mathfrak{F}=-\frac{1}{2 \pi i} \int d \epsilon f(\epsilon) \operatorname{Tr}\left(S^{\dagger} \frac{\partial S}{\partial \xi}\right),
$$

where $f(\epsilon)$ is the Fermi-Dirac distribution function with chemical potential $\mu$. This established result will be reproduced and generalized to the description of dissipation and fluctuations below.

\section{B. Gilbert damping as energy pumping}

Here we interpret Gilbert damping as an energy-pumping process by equating the results for energy dissipation from the microscopic adiabatic pumping formalism with the LLG phenomenology in terms of collective coordinates, Eq. (9). The adiabatic energy-loss rate of a scattering region in terms of a scattering matrix at zero temperature has been derived in Refs. 32 and 33. In the Appendixes, we present an alternative way to obtain this result at finite temperatures: ${ }^{34}$

$$
\dot{E}=\frac{\hbar}{4 \pi} \int d \epsilon\left(-\frac{\partial f}{\partial \epsilon}\right) \operatorname{Tr}\left[\frac{\partial S(\epsilon, t)}{\partial t} \frac{\partial S^{\dagger}(\epsilon, t)}{\partial t}\right] .
$$

Since we employ the adiabatic approximation, $S(\epsilon, t)$ is the energy-dependent scattering matrix for an instantaneous ("frozen") scattering potential at time $t$. In a magnetic system, the time dependence arises from its magnetization dynamics, $S(\epsilon, t)=S[\mathbf{m}(t)](\epsilon)$. In terms of the collective coordinates $\boldsymbol{\xi}(t), S(\epsilon, t)=S(\epsilon,\{\xi(t)\})$,

$$
\frac{\partial S[\mathbf{m}(t)]}{\partial t} \approx \sum_{a} \frac{\partial S}{\partial \xi_{a}} \dot{\xi}_{a},
$$

where the approximate sign has been discussed in the previous section. We can now identify the dissipation tensor (10) in terms of the scattering matrix

$$
\Gamma_{a b}=\frac{\hbar}{4 \pi} \int d \epsilon\left(-\frac{\partial f}{\partial \epsilon}\right) \operatorname{Tr}\left[\frac{\partial S(\epsilon)}{\partial \xi_{a}} \frac{\partial S^{\dagger}(\epsilon)}{\partial \xi_{b}}\right] .
$$

In the macrospin model, the Gilbert damping tensor can then be expressed as

$$
\tilde{\alpha}_{i j}=\frac{\gamma \hbar}{4 \pi M_{s}} \int d \epsilon\left(-\frac{\partial f}{\partial \epsilon}\right) \operatorname{Tr}\left[\frac{\partial S(\epsilon)}{\partial m_{i}} \frac{\partial S^{\dagger}(\epsilon)}{\partial m_{j}}\right],
$$

where $m_{i}$ is a Cartesian component of the magnetization direction.

\section{Gilbert damping and fluctuation-dissipation theorem}

At finite temperatures, the forces acting on the magnetization contain thermal fluctuations that are related to the Gilbert dissipation by the fluctuation-dissipation theorem, Eq. (14). The dissipation tensor is therefore accessible via the stochastic forces in thermal equilibrium.

The time dependence of the force operators

$$
\hat{\mathfrak{F}}(t)=-\frac{\partial \hat{H}_{\mathrm{ALDA}}(\mathbf{m})}{\partial \boldsymbol{\xi}}
$$

is caused by the thermal fluctuations of the magnetization. It is convenient to rearrange the Hamiltonian $\hat{H}_{\mathrm{ALDA}}$ into an unperturbed part that does not depend on the magnetization and a scattering potential $\hat{H}_{\mathrm{ALDA}}(\mathbf{m})=\hat{H}_{0}+\hat{V}(\mathbf{m})$. On the basis of scattering wave functions of the leads, the force operator reads

$$
\hat{\mathfrak{F}}=-\int d \epsilon \int d \epsilon^{\prime}\left\langle\epsilon \alpha\left|\frac{\partial \hat{V}}{\partial \xi}\right| \epsilon^{\prime} \beta\right\rangle \hat{a}_{\alpha}^{\dagger}(\epsilon) \hat{a}_{\beta}\left(\epsilon^{\prime}\right) e^{i\left(\epsilon-\epsilon^{\prime}\right) t / \hbar},
$$


where $\hat{a}_{\beta}$ annihilates an electron incident on the scattering region, $\beta$ labels the lead (left or right) and quantum numbers of the waveguide mode, and $\left|\epsilon^{\prime} \beta\right\rangle$ is an associated scattering eigenstate at energy $\epsilon^{\prime}$. We take again the left and right reservoirs to be in thermal equilibrium with the same chemical potentials, such that the expectation values

$$
\left\langle\hat{a}_{\alpha}^{\dagger}(\epsilon) \hat{a}_{\beta}\left(\epsilon^{\prime}\right)\right\rangle=\delta_{\alpha \beta} \delta\left(\epsilon-\epsilon^{\prime}\right) f(\epsilon) .
$$

The relation between the matrix element of the scattering potential and the $S$ matrix

$$
\left[S^{\dagger}(\epsilon) \frac{\partial S(\epsilon)}{\partial \xi}\right]_{\alpha \beta}=-2 \pi i\left\langle\epsilon \alpha\left|\frac{\partial \hat{V}}{\partial \xi}\right| \epsilon \beta\right\rangle
$$

follows from the relation derived in Eq. (61) below as well as unitarity of the $S$ matrix, $S^{\dagger} S=1$. Taking these relations into account, the expectation value of $\hat{\mathfrak{F}}$ is found to be Eq. (37). We now consider the fluctuations in the force $\hat{\mathfrak{f}}(t)=\hat{\mathfrak{F}}(t)-\langle\hat{\mathfrak{F}}(t)\rangle$, which involves expectation values

$$
\begin{aligned}
& \left\langle\hat{a}_{\alpha_{1}}^{\dagger}\left(\epsilon_{1}\right) \hat{a}_{\beta_{1}}\left(\epsilon_{1}^{\prime}\right) \hat{a}_{\alpha_{2}}^{\dagger}\left(\epsilon_{2}\right) \hat{a}_{\beta_{2}}\left(\epsilon_{2}^{\prime}\right)\right\rangle-\left\langle\hat{a}_{\alpha_{1}}^{\dagger}\left(\epsilon_{1}\right) \hat{a}_{\beta_{1}}\left(\epsilon_{1}^{\prime}\right)\right\rangle\left\langle\hat{a}_{\alpha_{2}}^{\dagger}\left(\epsilon_{2}\right) \hat{a}_{\beta_{2}}\left(\epsilon_{2}^{\prime}\right)\right\rangle \\
& =\delta_{\alpha_{1} \beta_{2}} \delta\left(\epsilon_{1}-\epsilon_{2}^{\prime}\right) \delta_{\beta_{1} \alpha_{2}} \delta\left(\epsilon_{1}^{\prime}-\epsilon_{2}\right) f\left(\epsilon_{1}\right)\left[1-f\left(\epsilon_{2}\right)\right],
\end{aligned}
$$

where we invoked Wick's theorem. Putting everything together, we finally find

$$
\left\langle\mathfrak{f}_{a}(t) \mathfrak{f}_{b}\left(t^{\prime}\right)\right\rangle=2 k_{B} T \delta\left(t-t^{\prime}\right) \Gamma_{a b},
$$

where $\Gamma_{a b}$ has been defined in Eq. (40). Comparing with Eq. (14), we conclude that the dissipation tensor $\Gamma_{a b}$ governing the fluctuations is identical to the one obtained from the energy pumping, Eq. (40), thereby confirming the fluctuation-dissipation theorem. In small ferromagnets, magnetization dissipation is dominated by spin-pumping to adjacent conductors, ${ }^{10}$ and a one-to-one correspondence between the spin-pumping enhanced dissipation and the spin-current fluctuations can be found that obeys the fluctuation-dissipation theorem of Eq. (14). ${ }^{35}$

\section{KUBO FORMULA}

Adiabatic magnetization dynamics perturbs the system weakly, thus allowing for a linear-response treatment of the instantaneous dissipation. In the present section, we demonstrate that the damping obtained from linear-response (Kubo) theory agrees ${ }^{36}$ with that of the scattering theory of magnetization dissipation in this limit. At sufficiently low temperatures or strong elastic disorder scattering, the coupling to phonons may be disregarded and is not discussed here.

The energy dissipation can be written as

$$
\dot{E}=\left\langle\frac{d \hat{H}}{d t}\right\rangle
$$

where \langle\rangle denotes the expectation value for the nonequilibrium state. We are interested in the adiabatic response of the system to a time-dependent perturbation. In the adiabatic (slow) regime, we can at any time expand the Hamiltonian around a static configuration at the reference time $t=0$,

$$
\hat{H}=\hat{H}_{\mathrm{st}}+\sum_{a} \delta \xi_{a}(t)\left(\frac{\partial \hat{H}}{\partial \xi_{a}}\right)_{\mathbf{m}(\mathbf{r}) \rightarrow \mathbf{m}_{\mathrm{st}}(\mathbf{r})} .
$$

The static part, $\hat{H}_{\text {st }}$, is the Hamiltonian for a magnetization for a fixed and arbitrary initial texture $\mathbf{m}_{\text {st }}$, as, without loss of generality, described by the collective coordinates $\xi_{a}$. Since we assume that the variation of the magnetization in time is small, a linear expansion in terms of the small deviations of the collective coordinate $\delta \xi_{i}(t)$ is valid for sufficiently short time intervals. We can then employ the Kubo formalism and express the energy dissipation as

$$
\dot{E}=\sum_{a} \delta \dot{\xi}_{a}(t)\left(\frac{\partial \hat{H}}{\partial \xi_{a}}\right)_{\mathbf{m}(\mathbf{r}) \rightarrow \mathbf{m}_{\mathrm{st}}(\mathbf{r})},
$$

where the expectation value of the out-of-equilibrium conservative force

$$
\left(\frac{\partial \hat{H}}{\partial \xi_{a}}\right)_{\mathbf{m}(\mathbf{r}) \rightarrow \mathbf{m}_{\mathrm{st}}(\mathbf{r})} \equiv \partial_{a} \hat{H}
$$

consists of an equilibrium contribution and a term linear in the perturbed magnetization direction:

$$
\left\langle\partial_{a} \hat{H}\right\rangle(t)=\left\langle\partial_{a} \hat{H}\right\rangle_{\mathrm{st}}+\sum_{b} \int_{-\infty}^{\infty} d t^{\prime} \chi_{a b}\left(t-t^{\prime}\right) \delta \xi_{b}\left(t^{\prime}\right) .
$$

Here, we introduced the retarded susceptibility

$$
\chi_{a b}\left(t-t^{\prime}\right)=-\frac{i}{\hbar} \theta\left(t-t^{\prime}\right)\left\langle\left[\partial_{a} \hat{H}(t), \partial_{b} \hat{H}\left(t^{\prime}\right)\right]\right\rangle_{\mathrm{st}},
$$

where \langle\rangle$_{\mathrm{st}}$ is the expectation value for the wave functions of the static configuration. Focusing on slow modulations, we can further simplify the expression by expanding

$$
\delta \xi_{a}\left(t^{\prime}\right) \approx \delta \xi_{a}(t)+\left(t^{\prime}-t\right) \delta \dot{\xi}_{a}(t),
$$

so that

$$
\begin{aligned}
\left\langle\partial_{a} \hat{H}\right\rangle= & \left\langle\partial_{a} \hat{H}\right\rangle_{\mathrm{st}}+\int_{-\infty}^{\infty} d t^{\prime} \chi_{a b}\left(t-t^{\prime}\right) \delta \xi_{b}(t) \\
& +\int_{-\infty}^{\infty} d t^{\prime} \chi_{a b}\left(t-t^{\prime}\right)\left(t^{\prime}-t\right) \delta \dot{\xi}_{b}(t) .
\end{aligned}
$$

The first two terms in this expression, $\left\langle\partial_{a} \hat{H}\right\rangle_{\mathrm{st}}+$ $\int_{-\infty}^{\infty} d t^{\prime} \chi_{a b}\left(t-t^{\prime}\right) \delta \xi_{b}(t)$, correspond to the energy variation with respect to a change in the static magnetization. These terms do not contribute to the dissipation since the magnetic excitations are transverse, $\mathbf{m} \cdot \mathbf{m}=\mathbf{0}$. Only the last term in Eq. (55) gives rise to dissipation. Hence, the energy loss reduces to 37

$$
\dot{E}=\left.i \sum_{i j} \delta \dot{\xi}_{a} \delta \dot{\xi}_{b} \frac{\partial \chi_{a b}^{S}}{\partial \omega}\right|_{\omega=0}
$$

where $\chi_{a b}^{S}(\omega)=\int_{-\infty}^{\infty} d t\left[\chi_{a b}(t)+\chi_{b a}(t)\right] e^{i \omega t} / 2$. The symmetrized susceptibility can be expanded as

$$
\chi_{a b}^{S}=\sum_{n m} \frac{\left(f_{n}-f_{m}\right)}{2} \frac{\left\langle n\left|\partial_{a} \hat{H}\right| m\right\rangle\left\langle m\left|\partial_{b} \hat{H}\right| n\right\rangle+(a \leftrightarrow b)}{\hbar \omega+i \eta-\left(\epsilon_{n}-\epsilon_{m}\right)},
$$

where $|n\rangle$ is an eigenstate of the Hamiltonian $\hat{H}_{\text {st }}$ with eigenvalue $\epsilon_{n}, f_{n} \equiv f\left(\epsilon_{n}\right), f(\epsilon)$ is the Fermi-Dirac distribution 
function at energy $\epsilon$, and $\eta$ is a positive infinitesimal constant. Therefore,

$$
\begin{aligned}
i\left(\frac{\partial \chi_{a b}^{S}}{\partial \omega}\right)_{\omega=0}= & \pi \sum_{n m}\left(-\frac{\partial f_{n}}{\partial \epsilon}\right)\left\langle n\left|\partial_{a} \hat{H}\right| m\right\rangle \\
& \times\left\langle m\left|\partial_{b} \hat{H}\right| n\right\rangle \delta\left(\epsilon_{n}-\epsilon_{m}\right),
\end{aligned}
$$

and the dissipation tensor

$$
\Gamma_{a b}=\pi \sum_{n m}\left(-\frac{\partial f_{n}}{\partial \epsilon}\right)\left\langle n\left|\partial_{a} \hat{H}\right| m\right\rangle\left\langle m\left|\partial_{b} \hat{H}\right| n\right\rangle \delta\left(\epsilon_{n}-\epsilon_{m}\right) .
$$

We now demonstrate that the dissipation tensor obtained from the Kubo linear-response formula, Eq. (59), is identical to the expression from scattering theory, Eq. (40), following the Fisher and Lee proof of the equivalence of linear response and scattering theory for the conductance. ${ }^{38}$

The static Hamiltonian $\hat{H}_{\text {st }}(\xi)=\hat{H}_{0}+\hat{V}(\xi)$ can be decomposed into a free-electron part $\hat{H}_{0}=-\hbar^{2} \nabla^{2} / 2 m$ and a scattering potential $\hat{V}(\xi)$. The eigenstates of $\hat{H}_{0}$ are denoted $\left|\varphi_{s, q}(\epsilon)\right\rangle$, with eigenenergies $\epsilon$, where $s= \pm$ denotes the longitudinal propagation direction along the system (say, to the left or to the right), and $q$ is a transverse quantum number determined by the lateral confinement. The potential $\hat{V}(\xi)$ scatters the particles between the propagating states forward or backward. The outgoing $(+)$ and incoming $(-)$ scattering eigenstates of the static Hamiltonian $\hat{H}_{\text {st }}$ are written as $\left|\psi_{s, q}^{( \pm)}(\epsilon)\right\rangle$, which form another complete basis with orthogonality relations $\left\langle\psi_{s, q}^{( \pm)}(\epsilon) \mid \psi_{s^{\prime}, q^{\prime}}^{( \pm)}\left(\epsilon^{\prime}\right)\right\rangle=\delta_{s, s^{\prime}} \delta_{q, q^{\prime}} \delta\left(\epsilon-\epsilon^{\prime}\right) .{ }^{39}$ These wave functions can be expressed as $\left|\psi_{s, q}^{( \pm)}(\epsilon)\right\rangle=\left[1+\hat{G}_{\mathrm{st}}^{( \pm)} \hat{V}\right]\left|\varphi_{s, q}\right\rangle$, where the retarded $(+)$ and advanced $(-)$ Green's functions read $\hat{G}_{\mathrm{st}}^{( \pm)}(\epsilon)=\left(\epsilon \pm i \eta-\hat{H}_{\mathrm{st}}\right)^{-1}$. By expanding $\Gamma_{a b}$ on the basis of outgoing wave functions, $\left|\psi_{s, q}^{(+)}\right\rangle$, the energy dissipation (59) becomes

$$
\begin{aligned}
\Gamma_{a b}= & \pi \sum_{s q, s^{\prime} q^{\prime}} \int d \epsilon\left(-\frac{\partial f_{s, q}}{\partial \epsilon}\right) \\
& \times\left\langle\psi_{s, q}^{(+)}\left|\partial_{a} \hat{H}\right| \psi_{s^{\prime}, q^{\prime}}^{(+)}\right\rangle\left\langle\psi_{s^{\prime}, q^{\prime}}^{(+)}\left|\partial_{b} \hat{H}\right| \psi_{s, q}^{(+)}\right\rangle,
\end{aligned}
$$

where wave functions should be evaluated at the energy $\epsilon$.

Let us now compare this result, Eq. (60), to the direct scattering matrix expression for the energy dissipation, Eq. (40). The $S$-matrix operator can be written in terms of the $T$ matrix as $\hat{S}(\epsilon ; \xi)=1-2 \pi i \hat{T}(\epsilon ; \xi)$, where the $T$ matrix is defined recursively by $\hat{T}=\hat{V}\left[1+\hat{G}_{\text {st }}^{(+)} \hat{T}\right]$. We then find

$$
\frac{\partial \hat{T}}{\partial \xi_{a}}=\left[1+\hat{V} \hat{G}_{\mathrm{st}}^{(+)}\right] \partial_{a} \hat{H}\left[1+\hat{G}_{\mathrm{st}}^{(+)} \hat{V}\right] .
$$

The change in the scattering matrix appearing in Eq. (40) is then

$$
\begin{aligned}
\frac{\partial S_{s^{\prime} q^{\prime}, s q}}{\partial \xi_{a}} & =-2 \pi i\left\langle\varphi_{s, q}\left|\left[1+\hat{V} \hat{G}_{\mathrm{st}}^{(+)}\right] \partial_{a} \hat{H}\left[1+\hat{G}_{\mathrm{st}}^{(+)} \hat{V}\right]\right| \varphi_{s^{\prime}, q^{\prime}}\right\rangle \\
& =-2 \pi i\left\langle\psi_{s^{\prime}, q^{\prime}}^{(-)}\left|\partial_{a} \hat{H}\right| \psi_{s^{\prime}, q^{\prime}}^{(+)}\right\rangle .
\end{aligned}
$$

Since

$$
\left\langle\psi_{s, q}^{(-)}(\epsilon)\right|=\sum_{s^{\prime} q^{\prime}} S_{s q, s^{\prime} q^{\prime}}\left\langle\psi_{s^{\prime} q^{\prime}}^{(+)}(\epsilon)\right|
$$

and $S S^{\dagger}=1$, we can write the linear-response result, Eq. (60), as energy pumping (40). This completes our proof of the equivalence between adiabatic energy pumping in terms of the $S$ matrix and the Kubo linear-response theory.

\section{CONCLUSIONS}

We have shown that most aspects of magnetization dynamics in ferromagnets can be understood in terms of the boundary conditions to normal metal contacts, i.e., a scattering matrix. By using the established numerical methods to compute electron transport based on scattering theory, this opens the way to compute dissipation in ferromagnets from first-principles. In particular, our formalism should work well for systems with strong elastic scattering due to a high density of large impurity potentials or in disordered alloys, including $\mathrm{Ni}_{1-x} \mathrm{Fe}_{x}(x=0.2$ represents the technologically important "permalloy").

The dimensionless Gilbert damping tensors (41) for macrospin excitations, which can be measured directly in terms of the broadening of the ferromagnetic resonance, have been evaluated for $\mathrm{Ni}_{1-x} \mathrm{Fe}_{x}$ alloys by ab initio methods. ${ }^{19}$ Permalloy is substitutionally disordered and damping is dominated by the spin-orbit interaction in combination with disorder scattering. Without adjustable parameters, good agreement has been obtained with the available low-temperature experimental data, which is a strong indication of the practical value of our approach.

In clean samples and at high temperatures, the electronphonon scattering importantly affects damping. Phonons are not explicitly included here, but the scattering theory of Gilbert damping can still be used for a frozen configuration of thermally displaced atoms, neglecting the inelastic aspect of scattering. ${ }^{13}$

While the energy pumping by scattering theory has been applied to described magnetization damping, ${ }^{12}$ it can be used to compute other dissipation phenomena. This has recently been demonstrated for the case of current-induced mechanical forces and damping, ${ }^{40}$ with a formalism analogous to that for current-induced magnetization torques. ${ }^{16,17}$

\section{ACKNOWLEDGMENTS}

We would like to thank Kjetil Hals, Paul J. Kelly, Yi Liu, Hans Joakim Skadsem, Anton Starikov, and Zhe Yuan for stimulating discussions, and M. Moskalets for pointing out Ref. 34. This work was supported by the EC Contract ICT-257159 "MACALO," the NSF under Grant No. DMR0840965, DARPA, FOM, DFG, and by the Project of Knowledge Innovation Program (PKIP) of the Chinese Academy of Sciences, Grant No. KJCX2.YW.W10

\section{APPENDIX A: ADIABATIC PUMPING}

Adiabatic pumping is the current response to a timedependent scattering potential to first order in the timevariation or "pumping" frequency when all reservoirs are at the same electrochemical potential. ${ }^{41}$ A compact formulation of the pumping charge current in terms of the instantaneous scattering matrix was derived in Ref. 42. In the same spirit, 
the energy current pumped out of the scattering region has been formulated (at zero temperature) in Ref. 33. Some time ago, we extended the charge pumping concept to include the spin degree of freedom and ascertained its importance in magnetoelectronic circuits. ${ }^{10}$ More recently, we demonstrated that the energy emitted by a ferromagnet with time-dependent magnetizations into adjacent conductors is not only caused by interface spin pumping, but also reflects the energy loss by spin-flip processes inside the ferromagnet ${ }^{12}$ and therefore Gilbert damping. Here we derive the energy pumping expressions at finite temperatures beyond the zero-temperature results derived in Ref. 33 and used in Ref. 12. Our results differ from an earlier extension to finite temperature derived in Ref. 43, and we point out the origin of the discrepancies, but they agree with the result in Eq. 103 of Ref. 34. The magnetization dynamics must satisfy the fluctuation-dissipation theorem, which is indeed the case in our formulation and in Ref. 34.

We proceed by deriving the charge, spin, and energy currents in terms of the time dependence of the scattering matrix of a two-terminal device. The transport direction is $x$ and the transverse coordinates are $\varrho=(y, z)$. An arbitrary single-particle Hamiltonian can be decomposed as

$$
H(\mathbf{r})=-\frac{\hbar^{2}}{2 m} \frac{\partial^{2}}{\partial x^{2}}+H_{\perp}(x, \varrho),
$$

where the transverse part is

$$
H_{\perp}(x, \varrho)=-\frac{\hbar^{2}}{2 m} \frac{\partial^{2}}{\partial \varrho^{2}}+V(x, \varrho) .
$$

$V(\varrho)$ is an elastic scattering potential in $2 \times 2$ Pauli spin space that includes the lattice, impurity, and self-consistent exchange-correlation potentials, including spin-orbit interaction and magnetic disorder. The scattering region is attached to perfect nonmagnetic electron waveguides (left $\alpha=L$ and right $\alpha=R$ ) with constant potential and without spin-orbit interaction. In lead $\alpha$, the transverse part of the $2 \times 2$ spinor wave function $\varphi_{\alpha}^{(n)}(x, \boldsymbol{\varrho})$ and its corresponding transverse energy $\epsilon_{\alpha}^{(n)}$ obey the Schrödinger equation

$$
H_{\perp}(\varrho) \varphi_{\alpha}^{(n)}(\varrho)=\epsilon_{\alpha}^{(n)} \varphi_{\alpha}^{(n)}(\varrho),
$$

where $n$ is the spin and orbit quantum number. These transverse waveguide modes form the basis for the expansion of the timedependent scattering states in lead $\alpha=L, R:{ }^{44}$

$$
\hat{\Psi}_{\alpha}=\int_{0}^{\infty} \frac{d k}{\sqrt{2 \pi}} \sum_{n \sigma} \varphi_{\alpha}^{(n)}(\varrho) e^{i \sigma k x} e^{-i \epsilon_{\alpha}^{(n k)} t / \hbar} \hat{c}_{\alpha}^{(n k \sigma)},
$$

where $\hat{c}_{\alpha}^{(n k \sigma)}$ annihilates an electron in mode $n$ incident $(\sigma=$ + ) or outgoing $(\sigma=-)$ in lead $\alpha$. The field operators satisfy the anticommutation relation

$$
\left\{\hat{c}_{\alpha}^{(n k \sigma)}, \hat{c}_{\beta}^{\dagger\left(n^{\prime} k^{\prime} \sigma^{\prime}\right)}\right\}=\delta_{\alpha \beta} \delta_{n n^{\prime}} \delta_{\sigma \sigma^{\prime}} \delta\left(k-k^{\prime}\right) .
$$

The total energy is $\epsilon_{\alpha}^{(n k)}=\hbar^{2} k^{2} / 2 m+\epsilon_{\alpha}^{(n)}$. In the leads, the particle, spins, and energy currents in the transport direction are

$$
\hat{I}^{(p)}=\frac{\hbar}{2 m i} \int d \varrho \operatorname{Tr}_{s}\left(\hat{\Psi}^{\dagger} \frac{\partial \hat{\Psi}}{\partial x}-\frac{\partial \hat{\Psi}^{\dagger}}{\partial x} \hat{\Psi}\right),
$$

$$
\begin{aligned}
& \hat{\boldsymbol{I}}^{(s)}=\frac{\hbar}{2 m i} \int d \varrho \operatorname{Tr}_{s}\left(\hat{\Psi}^{\dagger} \boldsymbol{\sigma} \frac{\partial \hat{\Psi}}{\partial x}-\frac{\partial \hat{\Psi}^{\dagger}}{\partial x} \boldsymbol{\sigma} \hat{\Psi}\right), \\
& \hat{\boldsymbol{I}}^{(e)}=\frac{\hbar}{4 m i} \int d \varrho \operatorname{Tr}_{s}\left(\hat{\Psi}^{\dagger} H \frac{\partial \hat{\Psi}}{\partial x}-\frac{\partial \hat{\Psi}^{\dagger}}{\partial x} H \hat{\Psi}\right)+\text { H.c. },
\end{aligned}
$$

where we suppressed the time $t$ and lead index $\alpha, \sigma=$ $\left(\sigma_{x}, \sigma_{y}, \sigma_{z}\right)$ is a vector of Pauli matrices, and $\operatorname{Tr}_{s}$ denotes the trace in spin space. Note that the spin current $\boldsymbol{I}_{s}$ flows in the $x$ direction with polarization vector $\boldsymbol{I}_{s} / I_{s}$. To avoid dependence on an arbitrary global potential shift, it is convenient to work with heat $\hat{I}^{(q)}$ rather than energy currents $\hat{I}^{(\epsilon)}$ :

$$
\hat{I}^{(q)}(t)=\hat{I}^{(\epsilon)}(t)-\mu \hat{I}^{(p)}(t),
$$

where $\mu$ is the chemical potential. Inserting the waveguide representation (A4) into (A5), the particle current reads ${ }^{44}$

$$
\begin{aligned}
\hat{I}_{\alpha}^{(p)}= & \frac{\hbar}{4 \pi m} \int_{0}^{\infty} d k d k^{\prime} \sum_{n \sigma \sigma^{\prime}}\left(\sigma k+\sigma^{\prime} k^{\prime}\right) \\
& \times e^{i\left(\sigma k-\sigma^{\prime} k^{\prime}\right) x} e^{-i\left[\epsilon_{\alpha}^{(n k)}-\epsilon_{\alpha}^{\left(n k^{\prime}\right)}\right] t / \hbar} \hat{c}_{\alpha}^{\dagger\left(n k^{\prime} \sigma^{\prime}\right)} \hat{c}_{\alpha}^{(n k \sigma)} .
\end{aligned}
$$

We are interested in the low-frequency limit of the Fourier transforms $I_{\alpha}^{(x)}(\omega)=\int_{-\infty}^{\infty} d t e^{i \omega t} I_{\alpha}^{(x)}(t)$. Following Ref. 44, we assume long wavelengths such that only the intervals with $k \approx k^{\prime}$ and $\sigma=\sigma^{\prime}$ contribute. In the adiabatic limit $\omega \rightarrow 0$, this approach is correct to leading order in $\hbar \omega / \epsilon_{F}$, where $\epsilon_{F}$ is the Fermi energy.By introducing the (current-normalized) operator

$$
\hat{c}_{\alpha}^{(n \sigma)}\left(\epsilon_{\alpha}^{(n k)}\right)=\frac{1}{\sqrt{\frac{d \epsilon_{\alpha}^{(n k \sigma)}}{d k}}} \hat{c}_{\alpha}^{(n k \sigma)},
$$

which obeys the anticommutation relations

$$
\left\{\hat{c}_{\alpha}^{(n \sigma)}\left(\epsilon_{\alpha}\right), \hat{c}_{\beta}^{\dagger\left(n^{\prime} \sigma^{\prime}\right)}\left(\epsilon_{\beta}\right)\right\}=\delta_{\alpha \beta} \delta_{n n^{\prime}} \delta_{\sigma \sigma^{\prime}} \delta\left(\epsilon_{\alpha}-\epsilon_{\beta}\right),
$$

the charge current can be written as

$$
\hat{I}_{\alpha}^{(c)}(t)=\frac{1}{2 \pi \hbar} \int_{\epsilon_{\alpha}^{(n)}}^{\infty} d \epsilon d \epsilon^{\prime} \sum_{n \sigma} \sigma e^{-i\left(\epsilon-\epsilon^{\prime}\right) t / \hbar} \hat{c}_{\alpha}^{\dagger(n \sigma)}\left(\epsilon^{\prime}\right) \hat{c}_{\alpha}^{(n \sigma)}(\epsilon) .
$$

We operate in the linear-response regime in which applied voltages and temperature differences as well as the externally induced dynamics disturb the system only weakly. Transport is then governed by states close to the Fermi energy. We may therefore extend the limits of the energy integration in Eq. (A10) from $\left(\epsilon_{\alpha}^{(n)}, \infty\right)$ to $(-\infty$ to $\infty)$. We relabel the annihilation operators so that $\hat{a}_{\alpha}^{(n k)}=\hat{c}_{\alpha+}^{(n k)}$ denotes particles incident on the scattering region from lead $\alpha$ and $\hat{b}_{\alpha}^{(n k)}=\hat{c}_{\alpha-}^{(n k)}$ denotes particles leaving the scattering region by lead $\alpha$. Using the Fourier transforms

$$
\begin{gathered}
\hat{c}_{\alpha}^{(n \sigma)}(\epsilon)=\int_{-\infty}^{\infty} d t \hat{c}_{\alpha}^{(n \sigma)}(t) e^{i \epsilon t / \hbar}, \\
\hat{c}_{\alpha}^{(n \sigma)}(t)=\frac{1}{2 \pi \hbar} \int_{-\infty}^{\infty} d \epsilon \hat{c}_{\alpha}^{(n \sigma)}(\epsilon) e^{-i \epsilon t / \hbar},
\end{gathered}
$$

we obtain in the low-frequency limit ${ }^{44}$

$$
\hat{I}_{\alpha}^{(p)}(t)=2 \pi \hbar\left[\hat{a}_{\alpha}^{\dagger}(t) \hat{a}_{\alpha}(t)-\hat{b}_{\alpha}^{\dagger}(t) \hat{b}_{\alpha}(t)\right],
$$


where $\hat{b}_{\alpha}$ is a column vector of the creation operators for all waveguide modes $\left\{\hat{b}_{\alpha}^{(n)}\right\}$. Analogous calculations lead to the spin current

$$
\hat{\boldsymbol{I}}_{\alpha}^{(s)}=2 \pi \hbar\left(\hat{a}_{\alpha}^{\dagger} \sigma \hat{a}_{\alpha}-\hat{b}_{\alpha}^{\dagger} \sigma \hat{b}_{\alpha}\right)
$$

and the energy current

$$
\hat{I}_{\alpha}^{(e)}=i \pi \hbar^{2}\left(\hat{a}_{\alpha}^{\dagger} \frac{\partial \hat{a}_{\alpha}}{\partial t}-\hat{b}_{\alpha}^{\dagger} \frac{\partial \hat{b}_{\alpha}}{\partial t}\right)+\text { H.c. }
$$

Next, we express the outgoing operators $\hat{b}(t)$ in terms of the incoming operators $\hat{a}(t)$ via the time-dependent scattering matrix (in the space spanned by all waveguide modes, including spin and orbit quantum number),

$$
\hat{b}_{\alpha}(t)=\sum_{\beta} \int d t^{\prime} S_{\alpha \beta}\left(t, t^{\prime}\right) \hat{a}_{\beta}\left(t^{\prime}\right) .
$$

When the scattering region is stationary, $S_{\alpha \beta}\left(t, t^{\prime}\right)$ only depends on the relative time difference $t-t^{\prime}$, and its Fourier transform with respect to the relative time is energy-independent, i.e., transport is elastic and can be computed for each energy separately. For time-dependent problems, $S_{\alpha \beta}\left(t, t^{\prime}\right)$ also depends on the total time $t+t^{\prime}$ and there is an inelastic contribution to transport as well. An electron can originate from a lead with energy $\epsilon$, pick up energy in the scattering region, and end up in the same or the other lead with different energy $\epsilon^{\prime}$.

The reservoirs are in equilibrium with controlled local chemical potentials and temperatures. We insert the $S$ matrix (A16) into the expressions for the currents, Eqs. (A13), (A14), and (A15), and use the expectation value at thermal equilibrium

$$
\left\langle\hat{a}_{\alpha}^{\dagger(n)}\left(t_{2}\right) \hat{a}_{\beta}^{(m)}\left(t_{1}\right)\right\rangle_{\mathrm{eq}}=\delta_{n m} \delta_{\alpha \beta} f_{\alpha}\left(t_{1}-t_{2}\right) / 2 \pi \hbar,
$$

where $f_{\beta}\left(t_{1}-t_{2}\right)=(2 \pi \hbar)^{-1} \int d \epsilon^{-i \epsilon\left(t_{1}-t_{2}\right) / \hbar} f_{\alpha}(\epsilon)$, and $f_{\alpha}(\epsilon)$ is the Fermi-Dirac distribution of electrons with energy $\epsilon$ in the $\alpha$ th reservoir. We then find

$$
\begin{aligned}
2 \pi \hbar\left\langle\hat{b}_{\alpha}^{\dagger}(t) \hat{b}_{\alpha}(t)\right\rangle_{\mathrm{eq}} & =\sum_{\beta} \int d t_{1} d t_{2} S_{\alpha \beta}^{*}\left(t, t_{2}\right) S_{\alpha \beta}\left(t, t_{1}\right) f_{\beta}\left(t_{1}-t_{2}\right), \\
2 \pi \hbar\left\langle\hat{b}_{\alpha}^{\dagger}(t) \sigma \hat{b}_{\alpha}(t)\right\rangle_{\mathrm{eq}} & =\sum_{\beta} \int d t_{1} d t_{2} S_{\alpha \beta}^{*}\left(t, t_{2}\right) \sigma S_{\alpha \beta}\left(t, t_{1}\right) f_{\beta}\left(t_{1}-t_{2}\right), \\
2 \pi \hbar\left\langle\hbar \partial_{t} \hat{b}_{\alpha}^{\dagger}(t) \hat{b}_{\alpha}(t)\right\rangle_{\mathrm{eq}} & =\sum_{\beta} \int d t_{1} d t_{2}\left[\hbar \partial_{t} S_{\alpha \beta}^{*}\left(t, t_{2}\right)\right] S_{\alpha \beta}\left(t, t_{1}\right) f_{\beta}\left(t_{1}-t_{2}\right) .
\end{aligned}
$$

Next, we use the Wigner representation (B1),

$$
S\left(t, t^{\prime}\right)=\frac{1}{2 \pi \hbar} \int_{-\infty}^{\infty} d \epsilon S\left(\frac{t+t^{\prime}}{2}, \epsilon\right) e^{-i \epsilon\left(t-t^{\prime}\right) / \hbar},
$$

and by Taylor expanding the Wigner represented $S$ matrix $S\left(\left(t+t^{\prime}\right) / 2, \epsilon\right)$ around $S(t, \epsilon), S\left(\left(t+t^{\prime}\right) / 2, \epsilon\right)=\sum_{n=0}^{\infty} \partial_{t}^{n} S(t, \epsilon)\left(t^{\prime}-\right.$ $t)^{n} /\left(2^{n} n !\right)$, we find

$$
S\left(t, t^{\prime}\right)=\frac{1}{2 \pi \hbar} \int_{-\infty}^{\infty} d \epsilon e^{-i \epsilon\left(t-t^{\prime}\right) / \hbar} e^{i \hbar \partial_{\epsilon} \partial_{t} / 2} S(t, \epsilon)
$$

and

$$
\hbar \partial_{t} S\left(t, t^{\prime}\right)=\frac{1}{2 \pi \hbar} \int_{-\infty}^{\infty} d \epsilon e^{-i \epsilon\left(t-t^{\prime}\right) / \hbar} e^{i \hbar \partial_{\epsilon} \partial_{t} / 2}\left(\frac{1}{2} \hbar \partial_{t}-i \epsilon\right) S(t, \epsilon) .
$$

The factor $1 / 2$ scaling the term $\hbar \partial_{t} S(t, \epsilon)$ arises from commuting $\epsilon$ with $e^{i \hbar \partial_{\epsilon} \partial_{t} / 2}$. The currents can now be evaluated as

$$
\begin{gathered}
I_{\alpha}^{(c)}(t)=-\frac{1}{2 \pi \hbar} \sum_{\beta} \int_{-\infty}^{\infty} d \epsilon\left\{\left[e^{-i \partial_{\epsilon} \partial_{t} \hbar / 2} S_{\beta \alpha}^{\dagger}(\epsilon, t)\right]\left[e^{i \partial_{\epsilon} \partial_{t} / 2 \hbar} S_{\alpha \beta}(\epsilon, t)\right] f_{\beta}(\epsilon)-f_{\alpha}(\epsilon)\right\}, \\
\mathbf{I}_{\alpha}^{(s)}(t)=-\frac{1}{2 \pi \hbar} \sum_{\beta} \int_{-\infty}^{\infty} d \epsilon\left\{\left[e^{-i \partial_{\epsilon} \partial_{t} \hbar / 2} S_{\beta \alpha}^{\dagger}(\epsilon, t)\right] \sigma\left[e^{i \partial_{\epsilon} \partial_{t} / 2 \hbar} S_{\alpha \beta}(\epsilon, t)\right] f_{\beta}(\epsilon)\right\}, \\
I_{\alpha}^{(\epsilon)}(t)=-\frac{1}{4 \pi \hbar} \sum_{\beta} \int_{-\infty}^{\infty} d \epsilon\left\{\left[e^{-i \partial_{\epsilon} \partial_{t} / 2 \hbar}\left(-i \hbar \partial_{t} / 2+\epsilon\right) S_{\beta \alpha}^{\dagger}(\epsilon, t)\right]\left[e^{+i \partial_{\epsilon} \partial_{t} / 2 \hbar} S_{\alpha \beta}(\epsilon, t)\right] f_{\beta}(\epsilon)-\epsilon f_{\alpha}(\epsilon)\right\} \\
-\frac{1}{4 \pi \hbar} \int_{-\infty}^{\infty} d \epsilon\left\{\left[e^{-i \partial_{\epsilon} \partial_{t} / 2 \hbar} S_{\beta \alpha}^{\dagger}(\epsilon, t)\right]\left[e^{i \partial_{\epsilon} \partial_{t} / 2 \hbar}\left(i \hbar \partial_{t} / 2+\epsilon\right) S_{\alpha \beta}(\epsilon, t)\right] f_{\beta}(\epsilon)-\epsilon f_{\alpha}(\epsilon)\right\},
\end{gathered}
$$

where the adjoint of the $S$ matrix has elements $S_{\beta \alpha}^{\dagger\left(n^{\prime}, n\right)}=S_{\alpha \beta}^{*\left(n, n^{\prime}\right)}$.

We are interested in the average (dc) currents, where simplified expressions can be found by partial integration over energy and time intervals. We will consider the total dc currents out of the scattering region, $I^{\text {(out) }}=-\sum_{\alpha} I_{\alpha}$, when the electrochemical 
potentials in the reservoirs are equal, $f_{\alpha}(\epsilon)=f(\epsilon)$ for all $\alpha$. The averaged pumped spin and energy currents out of the system in a time interval $\tau$ can be written compactly as

$$
\begin{gathered}
I_{\text {out }}^{(c)}=\frac{1}{2 \pi \hbar \tau} \int_{0}^{\tau} d t \int d \epsilon \operatorname{Tr}\left\{\left[f\left(\epsilon-\frac{i \hbar}{2} \frac{\partial}{\partial t}\right) S\right] S^{\dagger}-f(\epsilon)\right\}, \\
\mathbf{I}_{\text {out }}^{(s)}=\frac{1}{2 \pi \hbar \tau} \int_{0}^{\tau} d t \int d \epsilon \operatorname{Tr}\left\{\sigma\left[f\left(\epsilon-\frac{i \hbar}{2} \frac{\partial}{\partial t}\right) S\right] S^{\dagger}\right\}, \\
I_{\text {out }}^{(\epsilon)}=\frac{1}{2 \pi \hbar \tau} \int_{0}^{\tau} d t \int d \epsilon \operatorname{Tr}\left\{\left[\left(\epsilon-\frac{i \hbar}{2} \frac{\partial}{\partial t}\right) f\left(\epsilon-\frac{i \hbar}{2} \frac{\partial}{\partial t}\right) S\right] S^{\dagger}-\epsilon f(\epsilon)\right\} \\
+\frac{1}{2 \pi \hbar \tau} \int_{0}^{\tau} d t \int d \epsilon \operatorname{Tr}\left\{\left[f\left(\epsilon-\frac{i \hbar}{2} \frac{\partial}{\partial t}\right) S\right]\left(-i \hbar \frac{\partial S^{\dagger}}{\partial t}\right)\right\},
\end{gathered}
$$

where $\operatorname{Tr}$ is the trace over all waveguide modes (spin and orbital quantum numbers). As shown in Appendix C, the charge pumped into the reservoirs vanishes for a scattering matrix with a periodic time dependence when integrated over one cycle,

$$
I_{\mathrm{out}}^{(p)}=0
$$

This reflects particle conservation; the number of electrons cannot build up in the scattering region for periodic variations of the system. We can show that a similar contribution to the energy current, i.e., the first line in Eq. (A25c), vanishes, leading to the simple expression

$$
I_{\mathrm{out}}^{(e)}=-\frac{i}{2 \pi} \int_{0}^{\tau} \frac{d t}{\tau} \int d \epsilon \operatorname{Tr}\left\{\left[f\left(\epsilon-\frac{i \hbar}{2} \frac{\partial}{\partial t}\right) S\right] \frac{\partial S^{\dagger}}{\partial t}\right\} .
$$

Expanded to lowest order in the pumping frequency, the pumped spin current (A25b) becomes

$$
\mathbf{I}_{\text {out }}^{(s)}=\frac{1}{2 \pi \hbar} \int_{0}^{\tau} \frac{d t}{\tau} \int d \epsilon \operatorname{Tr}\left\{\left(S S^{\dagger} f-\frac{i \hbar}{2} \frac{\partial S}{\partial t} S^{\dagger} \partial_{\epsilon} f\right) \sigma\right\} .
$$

This formula is not the most convenient form to compute the current to specified order. $S S^{\dagger}$ also contains contributions that are linear and quadratic in the precession frequency since $S(t, \epsilon)$ is the $S$ matrix for a time-dependent problem. Instead, we would like to express the current in terms of the frozen scattering matrix $S_{\mathrm{fr}}(t, \epsilon)$. The latter is computed for an instantaneous, static electronic potential. In our case, this is determined by a magnetization configuration that depends parametrically on time: $S_{\mathrm{fr}}(t, \epsilon)=S[\mathbf{m}(t), \epsilon]$. Using unitarity of the time-dependent $S$ matrix (as elaborated in Appendix C), expanding it to lowest order in the pumping frequency, and inserting it into (A28) leads to ${ }^{42}$

$$
\mathbf{I}_{\text {out }}^{(s)}=\frac{i}{2 \pi} \sum_{\beta} \int_{0}^{\tau} \frac{d t}{\tau} \int d \epsilon\left(-\frac{\partial f}{\partial \epsilon}\right) \operatorname{Tr}\left\{\frac{\partial S_{\mathrm{fr}}}{\partial t} S_{\mathrm{fr}}^{\dagger} \boldsymbol{\sigma}\right\} .
$$

We evaluate the energy pumping by expanding (A27) to second order in the pumping frequency:

$$
I_{\text {out }}^{(e)}=\frac{\hbar}{4 \pi} \int_{0}^{\tau} \frac{d t}{\tau} \int d \epsilon \operatorname{Tr}\left\{-i f S \frac{\partial S^{\dagger}}{\partial t}-\left(\partial_{\epsilon} f\right) \frac{1}{2} \frac{\partial S}{\partial t} \frac{\partial S^{\dagger}}{\partial t}\right\} .
$$

As a consequence of unitarity of the $S$ matrix (see Appendix C), the first term vanishes to second order in the precession frequency: ${ }^{34}$

$$
I_{\mathrm{out}}^{(e)}=\frac{\hbar}{4 \pi} \int_{0}^{\tau} \frac{d t}{\tau} \int d \epsilon\left(-\frac{\partial f}{\partial \epsilon}\right) \operatorname{Tr}\left\{\frac{\partial S_{\mathrm{fr}}}{\partial t} \frac{\partial S_{\mathrm{fr}}^{\dagger}}{\partial t}\right\},
$$

where, at this point, we may insert the frozen scattering matrix since the current expression is already proportional to the square of the pumping frequency. Furthermore, since there is no net pumped charge current in one cycle (and we are assuming reservoirs in a common equilibrium), the pumped heat current is identical to the pumped energy current, $I_{\text {out }}^{(q)}=I_{\text {out }}^{(e)}$.

Our expression for the pumped energy current (A31) agrees with that derived in Ref. 33 at zero temperature and the one derived in Ref. 34 at finite temperatures. Our result (A31) differs from Ref. 43 at finite temperatures. The discrepancy can be explained as follows. Integration by parts over time $t$ in Eq. (A27), using

$$
\left[f\left(\epsilon-\frac{i \hbar}{2} \frac{\partial}{\partial t}\right) i \hbar \frac{\partial S}{\partial t}\right] S^{\dagger}=2\left[\epsilon f\left(\epsilon-\frac{i \hbar}{2} \frac{\partial}{\partial t}\right) S\right] S^{\dagger}-2\left[\left(\epsilon-\frac{i \hbar}{2} \frac{\partial}{\partial t}\right) f\left(\epsilon-\frac{i \hbar}{2} \frac{\partial}{\partial t}\right) S\right] S^{\dagger},
$$


and the unitarity condition from Appendix C,

$$
\int_{0}^{\tau} \frac{d t}{\tau} \int d \epsilon\left[\left(\epsilon-\frac{i \hbar}{2} \frac{\partial}{\partial t}\right) f\left(\epsilon-\frac{i \hbar}{2} \frac{\partial}{\partial t}\right) S\right] S^{\dagger}=\int_{0}^{\tau} \frac{d t}{\tau} \int d \epsilon \epsilon f(\epsilon),
$$

the dc pumped energy current can be rewritten as

$$
I_{\text {out }}^{(\epsilon)}=\frac{1}{\pi \hbar} \int_{0}^{\tau} \frac{d t}{\tau} \int d \epsilon \operatorname{Tr}\left\{\left[\epsilon f\left(\epsilon-\frac{i \hbar}{2} \frac{\partial}{\partial t}\right) S\right] S^{\dagger}-\epsilon f(\epsilon)\right\} .
$$

Next, we expand this to the second order in the pumping frequency and find

$$
I_{\text {out }}^{(\epsilon)}=\frac{1}{\pi \hbar} \int_{0}^{\tau} \frac{d t}{\tau} \int d \epsilon \operatorname{Tr}\left\{\epsilon f(\epsilon)\left(S S^{\dagger}-1\right)-\epsilon\left(\partial_{\epsilon} f\right) \frac{i \hbar}{2} \frac{\partial S}{\partial t} S^{\dagger}-\epsilon\left(\partial_{\epsilon}^{2} f\right) \frac{\hbar^{2}}{8} \frac{\partial^{2} S}{\partial t^{2}} S^{\dagger}\right\} .
$$

This form of the pumped energy current, Eq. (A35), agrees with Eq. (10) in Ref. 43 if one (incorrectly) assumes $S S^{\dagger}=1$. Although for the frozen scattering matrix, $S_{\mathrm{fr}} S_{\mathrm{fr}}^{\dagger}=1$, unitarity does not hold for the Wigner representation of the scattering matrix to the second order in the pumping frequency. $\left(S S^{\dagger}-1\right)$ therefore does not vanish but contributes to leading order in the frequency to the pumped current, which may not be neglected at finite temperatures. Only when this term is included is our new result Eq. (A31) recovered.

\section{APPENDIX B: FOURIER TRANSFORM AND WIGNER REPRESENTATION}

There is a long tradition in quantum theory to transform the two-time dependence of two-operator correlation functions such as scattering matrices by a mixed (Wigner) representation consisting of a Fourier transform over the time difference and an average time, which has distinct advantages when the scattering potential varies slowly in time. ${ }^{45}$ In order to establish conventions and notations, we present here a short exposure on how this works in our case.

The Fourier transform of the time-dependent annihilation operators is defined in Eqs. (A11) and (A12). Consider a function $A$ that depends on two times $t_{1}$ and $t_{2}, A=A\left(t_{1}, t_{2}\right)$. The Wigner representation with $t=\left(t_{1}+t_{2}\right) / 2$ and $t^{\prime}=t_{1}-$ $t_{2}$ is defined as

$$
\begin{aligned}
& A\left(t_{1}, t_{2}\right)=\frac{1}{2 \pi \hbar} \int_{-\infty}^{\infty} d \epsilon A(t, \epsilon) e^{-i \epsilon\left(t_{1}-t_{2}\right) / \hbar}, \\
& A(t, \epsilon)=\int_{-\infty}^{\infty} d t^{\prime} A\left(t+\frac{t^{\prime}}{2}, t-\frac{t^{\prime}}{2}\right) e^{i \epsilon t^{\prime} / \hbar} .
\end{aligned}
$$

We also need the Wigner representation of convolutions,

$$
(A \otimes B)\left(t_{1}, t_{2}\right)=\int_{-\infty}^{\infty} d t^{\prime} A\left(t_{1}, t^{\prime}\right) B\left(t^{\prime}, t_{2}\right) .
$$

By a series expansion, this can be expressed as ${ }^{45}$

$$
(A \otimes B)(t, \epsilon)=e^{-i\left(\partial_{t}^{A} \partial_{\epsilon}^{B}-\partial_{t}^{B} \partial_{\epsilon}^{A}\right) / 2} A(t, \epsilon) B(t, \epsilon),
$$

which we use in the following section.

\section{APPENDIX C: PROPERTIES OF THE $S$ MATRIX}

Here we discuss some general properties of the two-point time-dependent scattering matrix. Current conservation is reflected by the unitarity of the $S$ matrix, which can be expressed as

$$
\begin{aligned}
& \sum_{\beta n^{\prime} s^{\prime}} \int d t^{\prime} S_{n_{1} s_{1}, n^{\prime} s^{\prime}}^{\left(\alpha_{1} \beta\right)}\left(t_{1}, t^{\prime}\right) S_{n_{2} s_{2}, n^{\prime} s^{\prime}}^{\left(\alpha_{2} \beta\right) *}\left(t^{\prime}, t_{2}\right) \\
& \quad=\delta_{n_{1} n_{2}} \delta_{s_{1} s_{2}} \delta_{\alpha_{1} \alpha_{2}} \delta\left(t_{1}-t_{2}\right)
\end{aligned}
$$

Physically, this means that a particle entering the scattering region from a lead $\alpha$ at some time $t$ is bound to exit the scattering region in some lead $\beta$ at another (later) time $t^{\prime}$. Using Wigner representation (B1) and integrating over the local time variable, this implies [using Eq. (B4)]

$$
1=\left(S \otimes S^{\dagger}\right)(t, \epsilon)=e^{-i\left(\partial_{t}^{S} \partial_{\epsilon}^{S^{\dagger}}-\partial_{t}^{S^{\dagger}} \partial_{\epsilon}^{S}\right) / 2} S(t, \epsilon) S^{\dagger}(t, \epsilon),
$$

where 1 is a unit matrix in the space spanned by the waveguide modes (labeled by spin $s$ and orbital quantum number $n$ ). Similarly, we find

$$
1=\left(S^{\dagger} \otimes S\right)(t, \epsilon)=e^{+i\left(\partial_{t}^{S} \partial_{\epsilon}^{S^{\dagger}}-\partial_{t}^{S^{\dagger}} \partial_{\epsilon}^{S}\right) / 2} S^{\dagger}(t, \epsilon) S(t, \epsilon) .
$$

To second order in the precession frequency, subtracting and summing Eqs. (C2) and (C3), respectively, gives

$$
\operatorname{Tr}\left\{\frac{\partial S}{\partial t} \frac{\partial S^{\dagger}}{\partial \epsilon}-\frac{\partial S}{\partial \epsilon} \frac{\partial S^{\dagger}}{\partial t}\right\}=0
$$

and

$$
\operatorname{Tr}\left\{S S^{\dagger}-1\right\}=\operatorname{Tr}\left\{\frac{\partial^{2} S}{\partial t^{2}} \frac{\partial^{2} S^{\dagger}}{\partial \epsilon^{2}}-2 \frac{\partial^{2} S}{\partial t \partial \epsilon} \frac{\partial^{2} S^{\dagger}}{\partial t \partial \epsilon}+\frac{\partial^{2} S}{\partial \epsilon^{2}} \frac{\partial^{2} S^{\dagger}}{\partial t^{2}}\right\} .
$$

Furthermore, for any energy-dependent function $Z(\epsilon)$ and arbitrary matrix in the space spanned by spin and transverse waveguide modes $Y$, Eq. (C2) implies

$$
\begin{aligned}
& \frac{1}{\tau} \int_{0}^{\tau} d t \int d \epsilon Z(\epsilon) \operatorname{Tr}\left\{\left[e^{-i\left(\partial_{t}^{S} \partial_{\epsilon}^{S^{\dagger}}-\partial_{t}^{S^{\dagger}} \partial_{\epsilon}^{S}\right) / 2}\right.\right. \\
& \left.\left.\times S(t, \epsilon) S^{\dagger}(t, \epsilon)-1\right] Y\right\}=0 .
\end{aligned}
$$

Integration by parts with respect to $t$ and $\epsilon$ gives

$$
\begin{aligned}
& \frac{1}{\tau} \int_{0}^{\tau} d t \int d \epsilon \operatorname{Tr}\left\{\left[e^{-i\left(\partial_{t}^{S} \partial_{\epsilon}^{S \dagger}-\partial_{t}^{S} \partial_{\epsilon}^{Z S^{\dagger}}\right) / 2}\right.\right. \\
& \left.\left.\quad \times S(t, \epsilon) Z(\epsilon) S^{\dagger}(t, \epsilon)-Z(\epsilon)\right] Y\right\}=0,
\end{aligned}
$$


which can be simplified to

$$
\begin{aligned}
& \frac{1}{\tau} \int_{0}^{\tau} d t \int d \epsilon \operatorname{Tr} \\
& \quad \times\left\{\left(\left[Z\left(\epsilon+\frac{i}{2} \frac{\partial}{\partial t}\right) S(t, \epsilon)\right] S^{\dagger}(t, \epsilon)-Z(\epsilon)\right) Y\right\}=0 .
\end{aligned}
$$

Similarly from (C3), we find

$$
\begin{aligned}
& \frac{1}{\tau} \int_{0}^{\tau} d t \int d \epsilon \operatorname{Tr} \\
& \quad \times\left\{\left(S^{\dagger}(t, \epsilon)\left[Z\left(\epsilon-\frac{i}{2} \frac{\partial}{\partial t}\right) S(t, \epsilon)\right]-1\right) Y\right\}=0 .
\end{aligned}
$$

Using this result for $Y=1$ and $Z(\epsilon)=f(\epsilon)$ in the expression for the dc particle current (A25a), we see that unitarity indeed implies particle current conservation, $\sum_{\alpha} I_{\alpha}^{(c)}=0$, for a time-periodic potential. However, such a relation does not hold for spins. Choosing $Y=\sigma$, we cannot rewrite Eq. (C9) in the form (A25b) unless the $S$ matrix and the Pauli matrices commute. Unless the $S$ matrix is time- or spin-independent, a net spin current can be pumped out of the system, simultaneously exerting a torque on the scattering region.

Furthermore, choosing $Z(\epsilon)=\int_{0}^{\epsilon} d \epsilon^{\prime} f\left(\epsilon^{\prime}\right), \quad Y=1$, and expanding the difference between (C9) and (C8) to second order in frequency gives

$$
\frac{1}{\tau} \int_{0}^{\tau} d t \int d \epsilon \operatorname{Tr}\left\{f(\epsilon) \frac{\partial S(t, \epsilon)}{\partial t} S^{\dagger}(t, \epsilon)\right\}=0,
$$

which we use to eliminate the first term in the expression for the energy pumping, Eq. (A30).
${ }^{*}$ Arne.Brataas@ ntnu.no
${ }^{1}$ B. Heinrich, D. Fraitová, and V. Kamberský, Phys. Status Solidi 23, 501 (1967); V. Kambersky, Can. J. Phys. 48, 2906 (1970); V. Korenman and R. E. Prange, Phys. Rev. B 6, 2769 (1972); V. S. Lutovinov and M. Y. Reizer, ZETP 77, 707 (1979); V. L. Safonov and H. N. Bertram, Phys. Rev. B 61, R14893 (2000); J. Kunes and V. Kambersky, ibid. 65, 212411 (2002); V. Kambersky, ibid. 76, 134416 (2007); I. Garate and A. H. MacDonald, ibid. 79, 064404 (2009).

${ }^{2}$ For a review, see J. A. C. Bland and B. Heinrich, Ultrathin Magnetic Structures III: Fundamentals of Nanomagnetism (Springer-Verlag, Heidelberg, 2004), and references therein.

${ }^{3}$ For a review, see M. D. Stiles and J. Miltat, Top. Appl. Phys. 101, 225 (2006), and references therein.

${ }^{4}$ J. Foros, A. Brataas, Y. Tserkovnyak, and G. E. W. Bauer, Phys. Rev. B 78, 140402 (2008).

${ }^{5}$ S. Zhang and S. S.-L. Zhang, Phys. Rev. Lett. 102, 086601 (2009).

${ }^{6}$ C. H. Wong and Y. Tserkovnyak, Phys. Rev. B 80, 184411 (2009).

${ }^{7}$ W. F. Brown, Phys. Rev. 130, 1677 (1963).

${ }^{8}$ X. Waintal, E. B. Myers, P. W. Brouwer, and D. C. Ralph, Phys. Rev. B 62, 12317 (2000).

${ }^{9}$ A. Brataas, Yu. V. Nazarov, and G. E. W. Bauer, Phys. Rev. Lett. 84, 2481 (2000); A. Brataas, G. E. W. Bauer, and P. J. Kelly, Phys. Rep. 427, 157 (2006).

${ }^{10}$ Y. Tserkovnyak, A. Brataas, and G. E. W. Bauer, Phys. Rev. Lett. 88, 117601 (2002); Y. Tserkovnyak, A. Brataas, G. E. W. Bauer, and B. I. Halperin, Rev. Mod. Phys. 77, 1375 (2005).

${ }^{11}$ P. Bruno, Phys. Rev. B 52, 411 (1995).

${ }^{12}$ A. Brataas, Y. Tserkovnyak, and G. E. W. Bauer, Phys. Rev. Lett. 101, 037207 (2008).

${ }^{13}$ Y. Liu, Z. Yuan, A. A. Starikov, and P. J. Kelly, e-print arXiv:1102.5305.

${ }^{14}$ A. Brataas, Y. Tserkovnyak, G. E. W. Bauer, and B. I. Halperin, Phys. Rev. B 66, 060404 (2002); X. Wang, G. E. W. Bauer, B. J. van Wees, A. Brataas, and Y. Tserkovnyak, Phys. Rev. Lett. 97, 216602 (2006).

${ }^{15}$ B. Heinrich, Y. Tserkovnyak, G. Woltersdorf, A. Brataas, R. Urban, and G. E. W. Bauer, Phys. Rev. Lett. 90, 187601 (2003); M. V. Costache, M. Sladkov, S. M. Watts, C. H. van der Wal, and B. J. van Wees, ibid. 97, 216603 (2006); G. Woltersdorf, O. Mosendz,
B. Heinrich, and C. H. Back, ibid. 99, 246603 (2007).

${ }^{16}$ K. M. D. Hals, A. K. Nguyen, and A. Brataas, Phys. Rev. Lett. 102, 256601 (2009).

${ }^{17}$ K. M. D. Hals, Y. Tserkovnyak, and A. Bratas, Europhys. Lett. 90, 47002 (2010).

${ }^{18}$ K. Gilmore, Y. U. Idzerda, and M. D. Stiles, Phys. Rev. Lett. 99, 027204 (2007)

${ }^{19}$ A. A. Starikov, P. J. Kelly, A. Brataas, Y. Tserkovnyak, and G. E. W. Bauer, Phys. Rev. Lett. 105, 236601 (2010).

${ }^{20}$ H. Ebert, S. Mankovsky, D. Koedderitzsh, and P. J. Kelly, e-print arXiv:1102.4551.

${ }^{21}$ J. Kubler, Theory of Itinerant Electron Magnetism (Oxford University Press, New York, 2000).

${ }^{22}$ W. Kohn, Rev. Mod. Phys. 71, 1253 (1999).

${ }^{23}$ A. Zangwill and P. Soven, Phys. Rev. Lett. 45, 204 (1980).

${ }^{24}$ E. K. U. Gross and W. Kohn, Phys. Rev. Lett. 55, 2850 (1985).

${ }^{25}$ O. A. Tretiakov, D. J. Clarke, G. W. Chern, Ya. B. Bazaliy, and O. Tchernyshyov, Phys. Rev. Lett. 100, 127204 (2008).

${ }^{26}$ D. J. Clarke, O. A. Tretiakov, G. W. Chern, Ya. B. Bazaliy, and O. Tchernyshyov, Phys. Rev. B 78, 134412 (2008).

${ }^{27}$ A. Goussev, J. M. Robbins, and V. Slastikov, Phys. Rev. Lett. 104, 147202 (2010).

${ }^{28}$ N. L. Schryer and L. R. Walker, J. Appl. Phys. 45, 5466 (1974).

${ }^{29}$ Yu. V. Nazarov and Y. Blanter, Quantum Transport_Introduction to Nanoscience (Cambridge University Press, Cambridge, 2009).

${ }^{30}$ G. E. W. Bauer, Phys. Rev. Lett. 69, 1676 (1992).

${ }^{31}$ Y. Avishai and Y. B. Band, Phys. Rev. B 32, 2674 (1985).

${ }^{32}$ J. E. Avron, A. Elgart, G. M. Graf, and L. Sadun, Phys. Rev. Lett. 87, 236601 (2001).

${ }^{33}$ M. Moskalets and M. Büttiker, Phys. Rev. B 66, 035306 (2002); 66, 205320 (2002).

${ }^{34}$ L. Arrachea and M. Moskalets, Energy Transport and Heat Production in Quantum Engines, in Handbook of Nanophysics: Nanomedicine and Nanorobotics, edited by K. D. Sattler (CRC, Boca Raton, FL, 2010).

${ }^{35}$ J. Foros, A. Brataas, Y. Tserkovnyak, and G. E. W. Bauer, Phys. Rev. Lett. 95, 016601 (2005).

${ }^{36}$ G. D. Mahan, Many-particle Physics, 3rd ed. (Kluwer Academic, New York, 2010).

${ }^{37}$ E. Simanek and B. Heinrich, Phys. Rev. B 67, 144418 (2003). 
${ }^{38}$ D. S. Fisher and P. A. Lee, Phys. Rev. B 23, 6851 (1981).

${ }^{39}$ P. A. Mello and N. Kumar, Quantum Transport in Mesoscopic Systems (Oxford University Press, New York, 2005).

${ }^{40}$ S. D. Bennett, J. Maassen, and A. A. Clerk, Phys. Rev. Lett. 105, 217206 (2010); N. Bode, S. V. Kusminskiy, R. Egger, and F. von Oppen, e-print arXiv:1103.4809.
${ }^{41}$ M. Büttiker, H. Thomas, and A. Pretre, Z. Phys. B 94, 133 (1994).

${ }^{42}$ P. W. Brouwer, Phys. Rev. B 58, R10135 (1998).

${ }^{43}$ B. Wang and J. Wang, Phys. Rev. B 66, 125310 (2002).

${ }^{44}$ M. Büttiker, Phys. Rev. B 46, 12485 (1992).

${ }^{45}$ J. Rammer and H. Smith, Rev. Mod. Phys. 58, 323 (1985). 